\title{
Oscillation criteria for second-order nonlinear difference equations of Euler type
}

\section{Naoto Yamaoka*}

\section{*Correspondence:}

yamaoka@ms.osakafu-u.ac.jp Department of Mathematical Sciences, Osaka Prefecture University, Sakai, 599-8531, Japan

\section{Abstract}

The purpose of this paper is to present a pair of an oscillation theorem and a nonoscillation theorem for the second-order nonlinear difference equation

$$
\Delta^{2} x(n)+\frac{1}{n(n+1)} f(x(n))=0,
$$

where $f(x)$ is continuous on $\mathbb{R}$ and satisfies the signum condition $x f(x)>0$ if $x \neq 0$. The obtained results are best possible in a certain sense. Proof is given by means of the Riccati technique and phase plane analysis of a system. A discrete version of the Riemann-Weber generalization of Euler-Cauchy differential equation plays an important role in proving our results.

MSC: 39A12; 39A21

Keywords: oscillation constant; Euler-Cauchy equation; nonlinear difference equations; Riccati technique; phase plane analysis

\section{Introduction}

We consider the second-order nonlinear difference equation

$$
\Delta^{2} x(n)+\frac{1}{n(n+1)} f(x(n))=0, \quad n \geq n_{0},
$$

where $f(x)$ is a real-valued continuous function satisfying

$$
x f(x)>0 \quad \text { if } x \neq 0 .
$$

Here the forward difference operator $\Delta$ is defined as $\Delta x(n)=x(n+1)-x(n)$ and $\Delta^{2} x(n)=$ $\Delta(\Delta x(n))$.

A nontrivial solution $x(n)$ of (1.1) is said to be oscillatory if for every positive integer $N$ there exists $n \geq N$ such that $x(n) x(n+1) \leq 0$. Otherwise, it is said to be nonoscillatory, that is, the solution $x(n)$ is nonoscillatory if it is either eventually positive or eventually negative.

When $f(x)=\lambda x$, equation (1.1) becomes the linear difference equation

$$
\Delta^{2} x(n)+\frac{\lambda}{n(n+1)} x(n)=0,
$$


which is called the Euler-Cauchy difference equation. It is known that (1.3) has the general solution

$$
x(n)= \begin{cases}K_{1} \prod_{j=n_{0}}^{n-1}\left(1+\frac{z}{j}\right)+K_{2} \prod_{j=n_{0}}^{n-1}\left(1+\frac{1-z}{j}\right) & \text { if } \lambda \neq \frac{1}{4}, \\ \prod_{j=n_{0}}^{n-1}\left(1+\frac{1}{2 j}\right)\left\{K_{3}+K_{4} \sum_{k=n_{0}}^{n-1} \frac{2}{2 k+1}\right\} & \text { if } \lambda=\frac{1}{4},\end{cases}
$$

where $K_{1}, K_{2}, K_{3}, K_{4}$ are arbitrary constants and $z$ satisfies $z^{2}-z+\lambda=0$ (for the proof, see $[1-3])$. Hence, all nontrivial solutions of equation (1.3) are oscillatory if $\lambda>1 / 4$, and otherwise they are nonoscillatory (see the Appendix). In other words, $1 / 4$ is the lower bound for all nontrivial solutions of equation (1.3) to be oscillatory. Such a number is generally called the oscillation constant. Other results on the oscillation constant for difference equations can be found in [4-7] and the references cited therein.

Equation (1.3) is a discrete analogue of the Euler-Cauchy differential equation

$$
x^{\prime \prime}+\frac{\lambda}{t^{2}} x=0 .
$$

It is well known that an oscillation constant for equation (1.4) is also $1 / 4$ (see [8]). The oscillation constant for equation (1.4) plays an important role in the oscillation problem for linear, half-linear and nonlinear differential equations. For example, those results can be found in [8-15]. In particular, using phase plane analysis of Liénard system, Sugie and Kita [12] considered the second-order nonlinear differential equation

$$
x^{\prime \prime}+\frac{1}{t^{2}} f(x)=0
$$

and gave a pair of an oscillation theorem and a nonoscillation theorem (see [12, Theorems 3.1 and 4.1]). We note that their results are proved by using exact solutions of the Riemann-Weber version of Euler differential equation

$$
x^{\prime \prime}+\frac{1}{t^{2}}\left\{\frac{1}{4}+\frac{\lambda}{(\log t)^{2}}\right\} x=0 .
$$

By their results, we can show that an oscillation constant for equation (1.5) is $1 / 4$ provided

$$
f(x)=\left(\frac{1}{4}+\frac{\lambda}{\left(\log x^{2}\right)^{2}}\right) x
$$

for $|x|$ sufficiently large. A natural question now arises. What is an oscillation constant for equation (1.1) where $f(x)$ satisfies (1.6) for $|x|$ sufficiently large? The purpose of this paper is to answer the question. Our main results are stated as follows.

Theorem 1.1 Assume (1.2) and suppose that there exists $\lambda$ with $\lambda>1 / 4$ such that

$$
\frac{f(x)}{x} \geq \frac{1}{4}+\frac{\lambda}{\left(\log x^{2}\right)^{2}}
$$

for $|x|$ sufficiently large. Then all nontrivial solutions of equation (1.1) are oscillatory. 
Theorem 1.2 Assume (1.2) and suppose that

$$
\frac{f(x)}{x} \leq \frac{1}{4}+\frac{1}{4\left(\log x^{2}\right)^{2}}
$$

for $x>0$ or $x<0,|x|$ sufficiently large. Then equation (1.1) has a nonoscillatory solution.

Remark 1.1 As a discrete analogue of Euler-Cauchy differential equation (1.4), the linear difference equation

$$
\Delta^{2} x(n)+\frac{\lambda}{n(n+1)} x(n+1)=0
$$

is often considered instead of (1.3) (for example, see [4, 6, 7]), because Sturm's separation and comparison theorems can be applied to equation (1.9). However, it is not easy to find an exact solution of equation (1.9). On the other hand, equation (1.3) has the general solution, and therefore, we can get more precise information for discrete analogues of equation (1.4). In this paper, we consider the nonlinear term for equation (1.1) as $f(x(n))$ instead of $f(x(n+1))$ to use exact solutions of linear difference equations.

This paper is organized as follows. In Section 2, we give general solutions of a discrete version of the Riemann-Weber generalization of Euler differential equation and decide an oscillation constant for the discrete equation. In Section 3, we complete the proof of Theorem 1.1 by means of the Riccati technique. In Section 4, using phase plane analysis, we prove Theorem 1.2.

\section{General solutions of linear difference equations}

Consider the second-order linear difference equation

$$
\Delta^{2} x(n)+\frac{1}{n(n+1)}\left\{\frac{1}{4}+\frac{\lambda}{l(n) l(n+1)}\right\} x(n)=0,
$$

where the function $l(n)$ is positive and satisfies $\Delta l(n)=2 /(2 n+1)$. Note that $l(n) \sim \log n$ as $n \rightarrow \infty$. Here if $a(n)$ and $b(n)$ are positive functions, the notation $a(n) \sim b(n)$ as $n \rightarrow \infty$ means that $\lim _{n \rightarrow \infty} a(n) / b(n)=1$. Then we have the following result.

Proposition 2.1 Equation (2.1) has the general solution

$$
x(n)=\left\{\begin{array}{lr}
K_{1} \prod_{j=n_{0}}^{n-1}\left(1+\frac{1}{2 j}+\frac{z}{j l(j)}\right)+K_{2} \prod_{j=n_{0}}^{n-1}\left(1+\frac{1}{2 j}+\frac{1-z}{j l(j)}\right) & \text { if } \lambda \neq \frac{1}{4}, \\
\prod_{j=n_{0}}^{n-1}\left(1+\frac{1}{2 j}+\frac{1}{2 j l(j)}\right)\left\{K_{3}+K_{4} \sum_{k=n_{0}}^{n-1} \frac{2}{(2 k+1) l(k)+1}\right\} & \text { if } \lambda=\frac{1}{4},
\end{array}\right.
$$

where $K_{1}, K_{2}, K_{3}, K_{4}$ are arbitrary constants and $z$ is the root of the characteristic equation

$$
z^{2}-z+\lambda=0
$$


Proof Put

$$
\varphi(n)=\prod_{j=n_{0}}^{n-1}\left(1+\frac{1}{2 j}+\frac{z}{j l(j)}\right) \quad \text { and } \quad \psi(n)=\prod_{j=n_{0}}^{n-1}\left(1+\frac{1}{2 j}+\frac{1-z}{j l(j)}\right) .
$$

Then $\varphi(n)$ and $\psi(n)$ are the solutions of equation (2.1). We prove only the case that $\varphi(n)$ is a solution of equation (2.1), because the other case is carried out in the same manner. Here, we compute $\Delta \varphi(n)$ and $\Delta^{2} \varphi(n)$. Then we have

$$
\begin{aligned}
\Delta \varphi(n)= & \left.\frac{1}{2 n}+\frac{z}{n l(n)}\right) \varphi(n), \\
\Delta^{2} \varphi(n)= & \left\{\Delta\left(\frac{1}{2 n}\right)+\Delta\left(\frac{z}{n l(n)}\right)\right\} \varphi(n)+\left(\frac{1}{2(n+1)}+\frac{z}{(n+1) l(n+1)}\right) \Delta \varphi(n) \\
= & \left\{\frac{1}{2 n(n+1)}-\frac{z \Delta(n l(n))}{n(n+1) l(n) l(n+1)}\right\} \varphi(n) \\
& +\frac{1}{n+1}\left(\frac{1}{2}+\frac{z}{l(n+1)}\right) \frac{1}{n}\left(\frac{1}{2}+\frac{z}{l(n)}\right) \varphi(n) \\
= & -\frac{1}{n(n+1)}\left\{\frac{1}{2}+\frac{z\{l(n)+(n+1) \Delta l(n)\}}{l(n) l(n+1)}\right. \\
& \left.-\left(\frac{1}{4}+\frac{z}{2 l(n)}+\frac{z}{2 l(n+1)}+\frac{z^{2}}{l(n) l(n+1)}\right)\right\} \varphi(n) \\
= & -\frac{1}{n(n+1)}\left\{\frac{1}{4}+\frac{z\{2 l(n)+2(n+1) \Delta l(n)-l(n+1)-l(n)\}}{2 l(n) l(n+1)}-\frac{z^{2}}{l(n) l(n+1)}\right\} \varphi(n) \\
= & -\frac{1}{n(n+1)}\left\{\frac{1}{4}+\frac{z(2 n+1) \Delta l(n)}{2 l(n) l(n+1)}-\frac{z^{2}}{l(n) l(n+1)}\right\} \varphi(n) \\
= & -\frac{1}{n(n+1)}\left\{\frac{1}{4}+\frac{z-z^{2}}{l(n) l(n+1)}\right\} \varphi(n) .
\end{aligned}
$$

Since $z$ satisfies (2.2), $\varphi(n)$ is a solution of equation (2.1). We also see that $\varphi(n)$ and $\psi(n)$ are linearly independent if $\lambda \neq 1 / 4$. In fact, the Casoratian $W(n)$ of $\varphi(n)$ and $\psi(n)$ is given by

$$
W(n)=\operatorname{det}\left(\begin{array}{cc}
\varphi(n) & \psi(n) \\
\Delta \varphi(n) & \Delta \psi(n)
\end{array}\right)=\frac{1-2 z}{n l(n)} \varphi(n) \psi(n) \neq 0 .
$$

Hence, $K_{1} \varphi(n)+K_{2} \psi(n)$ is a general solution of (2.1).

We next consider the case that $\lambda=1 / 4$. Then (2.2) has the double root $1 / 2$. Hence, by a direct computation, we can show that

$$
\tilde{\varphi}(n)=\prod_{j=n_{0}}^{n-1}\left(1+\frac{1}{2 j}+\frac{1}{2 j l(j)}\right) \quad \text { and } \quad \tilde{\psi}(n)=\tilde{\varphi}(n) \sum_{k=n_{0}}^{n-1} \frac{2}{(2 k+1) l(k)+1}
$$

are linearly independent solutions of equation (2.1), and therefore, $K_{3} \tilde{\varphi}(n)+K_{4} \tilde{\psi}(n)$ is a general solution of (2.1).

To establish the oscillation constant for equation (2.1), we need the following lemma which is a corollary of the discrete l'Hospital rule (for example, see [16]). 
Lemma 2.1 Let $a(n)$ and $b(n)$ be defined for $n \geq n_{0}$. Suppose that $b(n)$ is positive and satisfies

$$
\sum_{n=n_{0}}^{\infty} b(n)=\infty
$$

If $a(n) \sim b(n)$ as $n \rightarrow \infty$, then

$$
\sum_{j=n_{0}}^{n} a(j) \sim \sum_{j=n_{0}}^{n} b(j)
$$

as $n \rightarrow \infty$.

Proposition 2.2 The oscillation constant for equation (2.1) is $1 / 4$. To be precise, equation (2.1) can be classified into two types as follows.

(i) If $\lambda>1 / 4$, then all nontrivial solutions of equation (2.1) are oscillatory.

(ii) If $\lambda \leq 1 / 4$, then all nontrivial solutions of equation (2.1) are nonoscillatory.

Proof We consider only the case that $\lambda \neq 1 / 4$ because the other case can be proved easily. In case $\lambda>1 / 4$, equation (2.2) has the conjugate roots $z=(1 \pm i \alpha) / 2$, where $\alpha=\sqrt{4 \lambda-1}$. Hence, by Proposition 2.1 and Euler's formula, the real solution of equation (2.1) can be written as

$$
x(n)=K_{5}\left(\prod_{j=n_{0}}^{n-1} r(j)\right) \cos \left(\sum_{j=n_{0}}^{n-1} \theta(j)\right)+K_{6}\left(\prod_{j=n_{0}}^{n-1} r(j)\right) \sin \left(\sum_{j=n_{0}}^{n-1} \theta(j)\right),
$$

where $r(j)$ and $\theta(j)$ satisfy $0<\theta(j)<\pi / 2$,

$$
r(j) \cos \theta(j)=1+\frac{1}{2 j}+\frac{1}{2 j l(j)} \quad \text { and } \quad r(j) \sin \theta(j)=\frac{\alpha}{2 j l(j)}
$$

for $n_{0} \leq j \leq n-1$. If $\left(K_{5}, K_{6}\right)=(0,0)$, then $x(n)$ is the trivial solution. On the other hand, if $\left(K_{5}, K_{6}\right) \neq(0,0)$, then

$$
x(n)=K_{7}\left(\prod_{j=n_{0}}^{n-1} r(j)\right) \sin \left(\sum_{j=n_{0}}^{n-1} \theta(j)+K_{8}\right),
$$

where $K_{7}=\sqrt{K_{5}^{2}+K_{6}^{2}}, \sin K_{8}=K_{5} / K_{7}$ and $\cos K_{8}=K_{6} / K_{7}$. Since

$$
\tan \theta(n)=\frac{\alpha}{2 n l(n)+l(n)+1} \rightarrow 0
$$

as $n \rightarrow \infty$, we obtain $\theta(n) \sim \tan \theta(n) \sim \alpha /(2 n l(n)) \sim \alpha /(2 n \log n)$ as $n \rightarrow \infty$. Using Lemma 2.1, we have

$$
\sum_{j=n_{0}}^{n-1} \theta(j) \sim \sum_{j=n_{0}}^{n-1} \frac{\alpha}{2 j \log j} \sim \frac{\alpha}{2} \log (\log n)
$$


as $n \rightarrow \infty$, because

$$
\sum_{j=n_{0}}^{n-1} \frac{\alpha}{2 j \log j} \sim \frac{\alpha}{2} \int_{n_{0}}^{n} \frac{d x}{x \log x} d x=\frac{\alpha}{2}\left\{\log (\log n)-\log \left(\log n_{0}\right)\right\} \rightarrow \infty
$$

as $n \rightarrow \infty$. We note that, for any sufficiently large $p \in \mathbb{N}$, there exists $n \in \mathbb{N}$ such that

$$
p \pi \leq \sum_{j=n_{0}}^{n-1} \theta(j)+K_{8}<(p+1) \pi
$$

because $\theta(n) \searrow 0$ as $n \rightarrow \infty$. Thus, we conclude that $x(n)$ is oscillatory.

We next consider the case that $\lambda<1 / 4$. Put

$$
\varphi(n)=\prod_{j=n_{0}}^{n-1}\left(1+\frac{1}{2 j}+\frac{z}{j l(j)}\right) \quad \text { and } \quad \psi(n)=\prod_{j=n_{0}}^{n-1}\left(1+\frac{1}{2 j}+\frac{1-z}{j l(j)}\right),
$$

where $z$ satisfies (2.2). Then, without loss of generality, we may assume that $z>1 / 2$. From Proposition 2.1, the solution of equation (2.1) can be represented as

$$
x(n)=K_{1} \varphi(n)+K_{2} \psi(n)=\varphi(n)\left\{K_{1}+K_{2} \frac{\psi(n)}{\varphi(n)}\right\}
$$

for some $K_{1} \in \mathbb{R}$ and $K_{2} \in \mathbb{R}$. Since

$$
\frac{\psi(n)}{\varphi(n)}=\prod_{j=n_{0}}^{n-1}\left(1-\frac{2 z-1}{j l(j)+l(j) / 2+z}\right) \leq \exp \left(-\sum_{j=n_{0}}^{n-1} \frac{2 z-1}{j l(j)+l(j) / 2+z}\right) \rightarrow 0
$$

as $n \rightarrow \infty$, we see that all nontrivial solutions of equation (2.1) are nonoscillatory.

\section{Oscillation theorem}

To begin with, we prepare some lemmas which are useful for proving oscillation criteria, Theorem 1.1.

Lemma 3.1 Assume (1.2) and suppose that equation (1.1) has a positive solution. Then the solution is increasing for $n$ sufficiently large and it tends to $\infty$ as $n \rightarrow \infty$.

Proof Let $x(n)$ be a positive solution of equation (1.1). Then there exists $n_{0} \in \mathbb{N}$ such that $x(n)>0$ for $n \geq n_{0}$. Hence, by (1.2) we have

$$
\Delta^{2} x(n)=-\frac{1}{n(n+1)} f(x(n))<0
$$

for $n \geq n_{0}$.

We first show that $\Delta x(t)>0$ for $n \geq n_{0}$. By way of contradiction, we suppose that there exists $n_{1} \geq n_{0}$ such that $\Delta x\left(n_{1}\right) \leq 0$. Then, using (3.1), we have $\Delta x(n)<\Delta x\left(n_{1}\right) \leq 0$ for $n>n_{1}$, and therefore, we can find $n_{2}>n_{1}$ such that $\Delta x\left(n_{2}\right)<0$. Using (3.1) again, we get $\Delta x(n) \leq \Delta x\left(n_{2}\right)<0$ for $n \geq n_{2}$. Hence, we obtain $x(n) \leq \Delta x\left(n_{2}\right)\left(n-n_{2}\right)+x\left(n_{2}\right) \rightarrow-\infty$ as 
$n \rightarrow \infty$, which is a contradiction to the assumption that $x(n)$ is positive for $n \geq n_{0}$. Thus, $x(n)$ is increasing for $n \geq n_{0}$.

We next suppose that $x(n)$ is bounded from above. Then there exists $L>0$ such that $\lim _{n \rightarrow \infty} x(n)=L$. Since $f(x)$ is continuous on $\mathbb{R}$, we have $\lim _{n \rightarrow \infty} f(x(n))=f(L)$, and therefore, there exists $n_{3} \geq n_{0}$ such that $0<f(L) / 2<f(x(n))$ for $n \geq n_{3}$. Hence, we have

$$
\Delta x(m)=\Delta x(n)+\sum_{j=m}^{n-1} \frac{1}{j(j+1)} f(x(j))>\frac{f(L)}{2} \sum_{j=m}^{n-1} \frac{1}{j(j+1)}=\frac{f(L)}{2}\left(\frac{1}{m}-\frac{1}{n}\right)
$$

for $n>m \geq n_{3}$. Taking the limit of this inequality as $n \rightarrow \infty$, we get $\Delta x(m) \geq f(L) / 2 m$ for $m \geq n_{3}$, and therefore, we obtain

$$
x(m) \geq x\left(n_{3}\right)+\frac{f(L)}{2} \sum_{k=n_{3}}^{m-1} \frac{1}{k} \rightarrow \infty
$$

as $m \rightarrow \infty$. This contradicts the assumption that $x(n)$ is bounded from above. Thus, we have $\lim _{n \rightarrow \infty} x(n)=\infty$. The proof is now complete.

Lemma 3.2 Suppose that the difference inequality

$$
\Delta w(n)+\frac{1}{n+w(n)}\left(w(n)-\frac{1}{2}\right)^{2} \leq 0
$$

has a positive solution. Then the solution is nonincreasing and tends to $1 / 2$ as $n \rightarrow \infty$.

Proof Let $w(n)$ be a positive solution of (3.2). Then there exists $n_{0} \in \mathbb{N}$ such that $w(n)>0$ for $n \geq n_{0}$. Hence, we see that $w(n)$ is nonincreasing because $w(n)$ satisfies

$$
\Delta w(n) \leq-\frac{1}{n+w(n)}\left(w(n)-\frac{1}{2}\right)^{2} \leq 0
$$

for $n \geq n_{0}$. Thus, we can find $\alpha \geq 0$ such that $w(n) \searrow \alpha$ as $n \rightarrow \infty$. If $\alpha \neq 1 / 2$, then there exists $n_{1} \geq n_{0}$ such that $|w(n)-1 / 2|>|\alpha-1 / 2| / 2$ for $n \geq n_{1}$. Since $w(n)$ is nonincreasing, there exists $n_{2} \geq n_{1}$ such that $w(n)<n$ for $n \geq n_{2}$. Hence, we have

$$
\Delta w(n) \leq-\frac{1}{n+w(n)}\left(w(n)-\frac{1}{2}\right)^{2} \leq-\frac{1}{2 n}\left(\frac{\alpha-1 / 2}{2}\right)^{2}
$$

for $n \geq n_{2}$, and therefore, we get

$$
w(n) \leq w\left(n_{2}\right)-\frac{1}{2}\left(\frac{\alpha-1 / 2}{2}\right)^{2} \sum_{j=n_{2}}^{n-1} \frac{1}{j} \rightarrow-\infty
$$

as $n \rightarrow \infty$. This is a contradiction to the assumption that $w(n)$ is positive for $n \geq n_{0}$.

We are now ready to prove Theorem 1.1.

Proof of Theorem 1.1 By way of contradiction, we suppose that equation (1.1) has a nonoscillatory solution $x(n)$. Then we may assume, without loss of generality, that $x(n)$ is 
eventually positive. Let $R$ be a large number satisfying the assumption (1.7) for $|x| \geq R$. From Lemma 3.1, $x(n)$ is increasing and $\lim _{n \rightarrow \infty} x(n)=\infty$, and therefore, there exists $n_{0} \in \mathbb{N}$ such that $x(n) \geq R$ and $\Delta x(n)>0$ for $n \geq n_{0}$.

We define

$$
w(n)=\frac{n \Delta x(n)}{x(n)} .
$$

Then, using (1.7), we have

$$
\begin{aligned}
\Delta w(n) & =\frac{\Delta(n \Delta x(n)) x(n)-n(\Delta x(n))^{2}}{x(n) x(n+1)} \\
& =\frac{\Delta x(n)+(n+1) \Delta^{2} x(n)}{x(n+1)}-n \frac{(\Delta x(n))^{2}}{x(n) x(n+1)} \\
& =\frac{\Delta x(n)-f(x(n)) / n}{x(n)} \frac{x(n)}{x(n+1)}-\frac{1}{n}\left(n \frac{\Delta x(n)}{x(n)}\right)^{2} \frac{x(n)}{x(n+1)} \\
& =\frac{1}{n}\left\{n \frac{\Delta x(n)}{x(n)}-\frac{f(x(n))}{x(n)}-\left(n \frac{\Delta x(n)}{x(n)}\right)^{2}\right\} \frac{x(n)}{x(n+1)} \\
& \leq \frac{1}{n}\left\{w(n)-\left(\frac{1}{4}+\frac{\lambda}{\left(\log x^{2}(n)\right)^{2}}\right)-w^{2}(n)\right\} \frac{x(n)}{x(n+1)} \\
& =-\frac{1}{n}\left\{\left(w(n)-\frac{1}{2}\right)^{2}+\frac{\lambda}{\left(\log x^{2}(n)\right)^{2}}\right\} \frac{x(n)}{x(n+1)} \\
& =-\frac{1}{n+w(n)}\left\{\left(w(n)-\frac{1}{2}\right)^{2}+\frac{\lambda}{\left(\log x^{2}(n)\right)^{2}}\right\}
\end{aligned}
$$

for $n \geq n_{0}$. From Lemma 3.2, we see that $w(n) \searrow 1 / 2$ as $n \rightarrow \infty$, because $w(n)$ is positive and satisfies (3.2) for $n \geq n_{0}$.

Since $\lambda>1 / 4$, we can find $\varepsilon_{0}>0$ such that

$$
\frac{1}{4}<\frac{1}{4}\left(1+4 \varepsilon_{0}\right)^{2}<\lambda
$$

Then we see that there exists $n_{1}>n_{0}$ such that $w(n) \leq 1 / 2+\varepsilon_{0}$ for $n \geq n_{1}$, that is, $x(n)$ satisfies

$$
\frac{x(n+1)}{x(n)} \leq\left\{1+\left(\frac{1}{2}+\varepsilon_{0}\right) \frac{1}{n}\right\}
$$

for $n \geq n_{1}$. Hence, we have

$$
\frac{x(n)}{x\left(n_{1}\right)}=\prod_{j=n_{1}}^{n-1} \frac{x(j+1)}{x(j)} \leq \prod_{j=n_{1}}^{n-1}\left\{1+\left(\frac{1}{2}+\varepsilon_{0}\right) \frac{1}{j}\right\}
$$

for $n \geq n_{1}$. Since $\log (1+z) \leq z$ for $z>-1$, we get

$$
\log x(n) \leq \sum_{j=n_{1}}^{n-1} \log \left\{1+\left(\frac{1}{2}+\varepsilon_{0}\right) \frac{1}{j}\right\}+\log x\left(n_{1}\right) \leq \sum_{j=n_{1}}^{n-1}\left(\frac{1}{2}+\varepsilon_{0}\right) \frac{1}{j}+\log x\left(n_{1}\right)
$$


for $n \geq n_{1}$. Recall that $l(n)$ satisfies $\Delta l(n)=2 /(2 n+1) \sim 1 / n$ as $n \rightarrow \infty$. Using Lemma 2.1, we see that

$$
l(n) \sim \sum_{j=n_{1}}^{n-1} \frac{1}{j}
$$

as $n \rightarrow \infty$. Hence, there exists $n_{2} \geq n_{1}$ such that

$$
\log x(n) \leq \frac{1+4 \varepsilon_{0}}{2} l(n) \leq \frac{1+4 \varepsilon_{0}}{2} l(n+1)
$$

for $n \geq n_{2}$. We therefore conclude that

$$
\Delta w(n) \leq-\frac{1}{n+w(n)}\left\{\left(w(n)-\frac{1}{2}\right)^{2}+\frac{\lambda}{\left(1+4 \varepsilon_{0}\right)^{2} l(n) l(n+1)}\right\}
$$

for $n \geq n_{2}$.

Let $v(n)$ be the function satisfying $v\left(n_{2}\right)=w\left(n_{2}\right)>0$ and $v(n+1)=F(n, v(n))$, where the function $F: \mathbb{N} \times[0, \infty) \rightarrow \mathbb{R}$ defined by

$$
F(n, v)=v-\frac{1}{n+v}\left\{\left(v-\frac{1}{2}\right)^{2}+\frac{\lambda}{\left(1+4 \varepsilon_{0}\right)^{2} l(n) l(n+1)}\right\} .
$$

Using mathematical induction on $n$, we show that the function $v(n)$ is well defined and satisfies $v(n) \geq w(n)>0$ for $n \geq n_{2}$. It is clear that the assertion is true for $n=n_{2}$. Assume that the assertion is true for $n=p$. Then $v(p+1)=F(p, v(p))$ exists because $v(p)>0$. Since

$$
\frac{d}{d v} F(p, v)=\frac{1}{(p+v)^{2}}\left\{\left(p+\frac{1}{2}\right)^{2}+\frac{\lambda}{\left(1+4 \varepsilon_{0}\right)^{2} l(p) l(p+1)}\right\} \geq 0,
$$

$F(p, v)$ is nondecreasing with respect to $v \in[0, \infty)$ for each fixed $p$. Hence, together with (3.4), we have

$$
v(p+1)=F(p, v(p)) \geq F(p, w(p)) \geq w(p+1)>0 .
$$

Thus, the assertion is also true for $n=p+1$.

Letting

$$
y(n)=\prod_{j=n_{2}}^{n-1}\left(1+\frac{v(j)}{j}\right),
$$

we can easily see that $y(n)$ is a positive solution of the difference equation

$$
\Delta^{2} y(n)+\frac{1}{n(n+1)}\left\{\frac{1}{4}+\frac{\lambda}{\left(1+4 \varepsilon_{0}\right)^{2} l(n) l(n+1)}\right\} y(n)=0 .
$$

Hence, from Proposition 2.2, we have

$$
\frac{\lambda}{\left(1+4 \varepsilon_{0}\right)^{2}} \leq \frac{1}{4}
$$

which is a contradiction to (3.3). 


\section{Nonoscillation theorem}

In this section, we give a sufficient condition for equation (1.1) to have a nonoscillatory solution. Let $x(n)$ be a solution of equation (1.1) and put $y(n)=n \Delta x(n)-x(n)$. Then we have

$$
\Delta y(n)=\Delta x(n)+(n+1) \Delta^{2} x(n)-\Delta x(n)=-\frac{1}{n} f(x(n)),
$$

and therefore, we can transform (1.1) into the system

$$
n \Delta x(n)=y(n)+x(n), \quad n \Delta y(n)=-f(x(n)) .
$$

To prove Theorem 1.2, we need the following results.

Lemma 4.1 Let $(x(n), y(n))$ be a nontrivial solution of system (4.1). If $(x(n), y(n)) \in D_{k}$, then $(x(n+1), y(n+1)) \in D_{k} \cup D_{k+1}$ for $k=1,2,3,4$, where

$$
\begin{array}{ll}
D_{1}=\left\{(x, y) \in \mathbb{R}^{2}: x>0, y \geq-x\right\}, & D_{2}=\left\{(x, y) \in \mathbb{R}^{2}: x>0, y<-x\right\}, \\
D_{3}=\left\{(x, y) \in \mathbb{R}^{2}: x<0, y \leq-x\right\}, & D_{4}=\left\{(x, y) \in \mathbb{R}^{2}: x<0, y>-x\right\}
\end{array}
$$

and $D_{5}=D_{1}$

Proof We prove only the case $k=1$, because the other cases are carried out in the same manner. Let $(x(n), y(n)) \in D_{1}$. Then we have $n \Delta x(n)=y(n)+x(n) \geq 0$, and therefore, we obtain $x(n+1) \geq x(n)$. Hence, we conclude that $(x(n+1), y(n+1)) \in D_{1} \cup D_{2}$.

Lemma 4.2 Suppose that $\theta(n)$ and $\varphi(n)$ satisfy $\theta\left(n_{0}\right)=\varphi\left(n_{0}\right)$ and

$$
\theta(n+1) \geq F(n, \theta(n)), \quad \varphi(n+1)=F(n, \varphi(n))
$$

for $n_{0} \leq n<n_{1}$, where $F(n, x)$ is nondecreasing with respect to $x \in \mathbb{R}$ for each fixed $n$. Then $\theta(n) \geq \varphi(n)$ for $n_{0} \leq n \leq n_{1}$.

Proof We use mathematical induction on $n$. It is clear that the assertion is true for $n=n_{0}$. Assume that $\theta(n) \geq \varphi(n)$ for $n=p<n_{1}$. Since $F(p, x)$ is nondecreasing with respect to $x$ for each fixed $p$, we have $\theta(p+1) \geq F(p, \theta(p)) \geq F(p, \varphi(p))=\varphi(p+1)$. Thus, the assertion is also true for $n=p+1$. This completes the proof.

We are now ready to prove Theorem 1.2.

Proof of Theorem 1.2 We give only the proof of the case that

$$
\frac{f(x)}{x} \leq \frac{1}{4}+\frac{1}{4\left(\log x^{2}\right)^{2}}
$$

for $x>0$ sufficiently large. The proof is by contradiction. Suppose that all nontrivial solutions of equation (1.1) are oscillatory. Let $(x(n), y(n))$ be the solution of system (4.1) satisfying the initial condition

$$
\left(x\left(n_{0}\right), y\left(n_{0}\right)\right)=\left(e^{l\left(n_{0}+1\right) / 2},\left(-\frac{1}{2}+\frac{1}{2 l\left(n_{0}\right)}\right) e^{l\left(n_{0}+1\right) / 2}\right) .
$$


Then $x(n)$ is a nontrivial oscillatory solution of equation (1.1) and

$$
\left(x\left(n_{0}\right), y\left(n_{0}\right)\right) \in\{(x, y): x>0, y \geq-x / 2\} \stackrel{\text { def }}{=} \tilde{D},
$$

and therefore, $(x(n), y(n))$ cannot stay in $\tilde{D} \subset D_{1}$. By Lemma 4.1, there exists $n_{1}>n_{0}$ such that

$$
(x(n), y(n)) \in \tilde{D} \quad \text { for } n_{0} \leq n<n_{1} \quad \text { and } \quad\left(x\left(n_{1}\right), y\left(n_{1}\right)\right) \in\{(x, y): y<-x / 2<0\} .
$$

Hence, we see that

$$
n \Delta x(n)=y(n)+x(n) \geq-\frac{x(n)}{2}+x(n)=\frac{x(n)}{2}
$$

for $n_{0} \leq n<n_{1}$, and therefore, we have $x(n+1) \geq\{1+1 /(2 n)\} x(n)$ for $n_{0} \leq n<n_{1}$. Thus, we get

$$
\frac{x(n)}{x\left(n_{0}\right)}=\prod_{j=n_{0}}^{n-1} \frac{x(j+1)}{x(j)} \geq \prod_{j=n_{0}}^{n-1}\left(1+\frac{1}{2 j}\right)
$$

for $n_{0} \leq n<n_{1}$. Hence, we obtain

$$
\begin{aligned}
\log x(n) & \geq \sum_{j=n_{0}}^{n-1} \log \left(1+\frac{1}{2 j}\right)+\log x\left(n_{0}\right)=\sum_{j=n_{0}}^{n-1} \log \left(1+\frac{1}{2 j}\right)+\frac{l\left(n_{0}+1\right)}{2} \\
& \geq \sum_{j=n_{0}}^{n-1}\left\{\frac{1}{2 j}-\frac{1}{2}\left(\frac{1}{2 j}\right)^{2}\right\}+\frac{l\left(n_{0}+1\right)}{2}=\frac{1}{2} \sum_{j=n_{0}}^{n-1}\left(\frac{1}{j}-\frac{1}{4 j^{2}}\right)+\frac{l\left(n_{0}+1\right)}{2} \\
& \geq \frac{1}{2} \sum_{j=n_{0}}^{n-1} \frac{2}{2 j+3}+\frac{l\left(n_{0}+1\right)}{2}=\frac{1}{2}\left\{\sum_{j=n_{0}}^{n-1} \Delta l(j+1)+l\left(n_{0}+1\right)\right\}=\frac{l(n+1)}{2} \geq \frac{l(n)}{2}
\end{aligned}
$$

for $n_{0} \leq n<n_{1}$. We define $\theta(n)=y(n) / x(n)$. Then, using (1.8), we have

$$
\begin{aligned}
\Delta \theta(n) & =\frac{(\Delta y(n)) x(n)-y(n) \Delta x(n)}{x(n) x(n+1)}=\frac{-f(x(n)) x(n)-y(n)(y(n)+x(n))}{n x(n) x(n+1)} \\
& =-\frac{x(n)}{n x(n+1)}\left\{\frac{f(x(n))}{x(n)}+\frac{y^{2}(n)}{x^{2}(n)}+\frac{y(n)}{x(n)}\right\} \\
& =-\frac{1}{\theta(n)+n+1}\left\{\theta^{2}(n)+\theta(n)+\frac{f(x(n))}{x(n)}\right\} \\
& \geq-\frac{1}{\theta(n)+n+1}\left\{\theta^{2}(n)+\theta(n)+\frac{1}{4}+\frac{1}{4\left(\log x^{2}(n)\right)^{2}}\right\} \\
& \geq-\frac{1}{\theta(n)+n+1}\left\{\left(\theta(n)+\frac{1}{2}\right)^{2}+\frac{1}{4 l(n) l(n+1)}\right\}
\end{aligned}
$$

for $n_{0} \leq n<n_{1}$. Note that $\theta(n)$ satisfies

$$
\theta\left(n_{0}\right)=\frac{x\left(n_{0}\right)}{y\left(n_{0}\right)}=-\frac{1}{2}+\frac{1}{2 l\left(n_{0}\right)} \quad \text { and } \quad \theta\left(n_{1}\right)<-\frac{1}{2} .
$$


We compare the function $\theta(n)$ with a solution

$$
\varphi(n)=-\frac{1}{2}+\frac{1}{2 l(n)}
$$

of the equation

$$
\Delta \varphi(n)=-\frac{1}{\varphi(n)+n+1}\left\{\left(\varphi(n)+\frac{1}{2}\right)^{2}+\frac{1}{4 l(n) l(n+1)}\right\} .
$$

It follows from Lemma 4.2 that $\varphi(n) \leq \theta(n)$ for $n_{0} \leq n \leq n_{1}$ because $\varphi\left(n_{0}\right)=\theta\left(n_{0}\right)$. Hence, together with (4.2) and (4.3), we have

$$
-\frac{1}{2}<\varphi\left(n_{1}\right) \leq \theta\left(n_{1}\right)<-\frac{1}{2}
$$

which is a contradiction. This completes the proof.

\section{Appendix: Euler-Cauchy difference equations}

In this appendix, we show that the oscillation constant for Euler-Cauchy difference equation (1.3) is $1 / 4$, that is, we prove the following result.

Proposition A.1 Equation (1.3) can be classified into two types as follows.

(i) If $\lambda>1 / 4$, then all nontrivial solutions of equation (1.3) are oscillatory.

(ii) If $\lambda \leq 1 / 4$, then all nontrivial solutions of equation (1.3) are nonoscillatory.

Proof The general solution of equation (1.3) is given by

$$
x(n)= \begin{cases}K_{1} \prod_{j=n_{0}}^{n-1}\left(1+\frac{z}{j}\right)+K_{2} \prod_{j=n_{0}}^{n-1}\left(1+\frac{1-z}{j}\right) & \text { if } \lambda \neq \frac{1}{4}, \\ \prod_{j=n_{0}}^{n-1}\left(1+\frac{1}{2 j}\right)\left\{K_{3}+K_{4} \sum_{k=n_{0}}^{n-1} \frac{2}{2 k+1}\right\} & \text { if } \lambda=\frac{1}{4},\end{cases}
$$

where $K_{1}, K_{2}, K_{3}, K_{4}$ are arbitrary constants and $z$ satisfies

$$
z^{2}-z+\lambda=0
$$

(for the proof, see [1-3]). Hence, we consider only the case that $\lambda \neq 1 / 4$ because we can easily check that all nontrivial solutions of equation (1.3) are nonoscillatory if $\lambda=1 / 4$.

In case $\lambda>1 / 4$, equation (A.2) has the conjugate roots $z=(1 \pm i \alpha) / 2$, where $\alpha=\sqrt{4 \lambda-1}$. Hence, by (A.1) and Euler's formula, the real solution of equation (1.3) can be written as

$$
x(n)=K_{5}\left(\prod_{j=n_{0}}^{n-1} r(j)\right) \cos \left(\sum_{j=n_{0}}^{n-1} \theta(j)\right)+K_{6}\left(\prod_{j=n_{0}}^{n-1} r(j)\right) \sin \left(\sum_{j=n_{0}}^{n-1} \theta(j)\right),
$$

where $r(j)$ and $\theta(j)$ satisfy $0<\theta(j)<\pi / 2$,

$$
r(j) \cos \theta(j)=1+\frac{1}{2 j} \text { and } \quad r(j) \sin \theta(j)=\frac{\alpha}{2 j}
$$


for $n_{0} \leq j \leq n-1$. If $\left(K_{5}, K_{6}\right) \neq(0,0)$, then

$$
x(n)=K_{7}\left(\prod_{j=n_{0}}^{n-1} r(j)\right) \sin \left(\sum_{j=n_{0}}^{n-1} \theta(j)+K_{8}\right),
$$

where $K_{7}=\sqrt{K_{5}^{2}+K_{6}^{2}}, \sin K_{8}=K_{5} / K_{7}$ and $\cos K_{8}=K_{6} / K_{7}$. Since

$$
\tan \theta(n)=\frac{\alpha}{2 n+1} \rightarrow 0
$$

as $n \rightarrow \infty$, we obtain $\theta(n) \sim \tan \theta(n)$ as $n \rightarrow \infty$. Using Lemma 2.1, we have

$$
\sum_{j=n_{0}}^{n-1} \theta(j) \sim \sum_{j=n_{0}}^{n-1} \frac{\alpha}{2 j+1} \sim \frac{\alpha}{2} \log n
$$

as $n \rightarrow \infty$. Hence, for any sufficiently large $p \in \mathbb{N}$, there exists $n \in \mathbb{N}$ such that

$$
p \pi \leq \sum_{j=n_{0}}^{n-1} \theta(j)+K_{8}<(p+1) \pi
$$

and therefore, $x(n)$ is oscillatory.

We next consider the case that $\lambda<1 / 4$. Put

$$
\varphi(n)=\prod_{j=n_{0}}^{n-1}\left(1+\frac{z}{j}\right) \quad \text { and } \quad \psi(n)=\prod_{j=n_{0}}^{n-1}\left(1+\frac{1-z}{j}\right)
$$

where $z$ satisfies (A.2). Then, without loss of generality, we may assume that $z>1 / 2$. From (A.1), the solution of equation (1.3) can be represented as

$$
x(n)=K_{1} \varphi(n)+K_{2} \psi(n)=\varphi(n)\left\{K_{1}+K_{2} \frac{\psi(n)}{\varphi(n)}\right\}
$$

for some $K_{1} \in \mathbb{R}$ and $K_{2} \in \mathbb{R}$. By Stirling's formula for the gamma function, we see that

$$
\Gamma(t+1) \sim \sqrt{2 \pi t}\left(\frac{t}{e}\right)^{t}
$$

as $t \rightarrow \infty$, where $\Gamma$ is the gamma function (as to Stirling's formula, for example, see [17]). Hence, we have

$$
\varphi(n)=\prod_{j=n_{0}}^{n-1}\left(1+\frac{z}{j}\right)=\frac{\Gamma\left(n_{0}\right) \Gamma(n+z)}{\Gamma\left(n_{0}+z\right) \Gamma(n)} \sim \frac{\Gamma\left(n_{0}\right)}{\Gamma\left(n_{0}+z\right)} n^{z} \quad \text { and } \quad \psi(n) \sim \frac{\Gamma\left(n_{0}\right)}{\Gamma\left(n_{0}+1-z\right)} n^{1-z}
$$

as $n \rightarrow \infty$, and therefore, we obtain

$$
\lim _{n \rightarrow \infty} \frac{\psi(n)}{\varphi(n)}=0
$$

Thus, we conclude that all nontrivial solutions of equation (1.3) are nonoscillatory. 


\section{Competing interests}

The author declares that they have no competing interests.

\section{Acknowledgements}

The author thanks the referees for their valuable suggestions that helped to improve this manuscript.

Received: 18 August 2012 Accepted: 30 November 2012 Published: 19 December 2012

\section{References}

1. Akin-Bohner, E, Bohner, M: Miscellaneous dynamic equations. Methods Appl. Anal. 10, 11-30 (2003)

2. Bohner, M, Peterson, A: Dynamic Equations on Time Scales. An Introduction with Applications. Birkhäuser, Boston (2001)

3. Huff, S, Olumolode, G, Pennington, N, Peterson, A: Oscillation of an Euler-Cauchy dynamic equation. Discrete Contin. Dyn. Syst., 423-431 (2003)

4. Došlý, O, Fišnarová, S: Linearized Riccati technique and (non-)oscillation criteria for half-linear difference equations. Adv. Differ. Equ. 2008, Art. ID 438130 (2008)

5. Došlý, O, Hilscher, R: A class of Sturm-Liouville difference equations: (non)oscillation constants and property BD. Comput. Math. Appl. 45, 961-981 (2003)

6. Luef, F, Teschl, G: On the finiteness of the number of eigenvalues of Jacobi operators below the essential spectrum. J. Differ. Equ. Appl. 10, 299-307 (2004)

7. Zhang, G, Cheng, SS: A necessary and sufficient oscillation condition for the discrete Euler equation. Panam. Math. J. 9, 29-34 (1999)

8. Swanson, CA: Comparison and Oscillation Theory of Linear Differential Equations. Academic Press, New York (1968)

9. Došlý, O, Hasil, P: Critical oscillation constant for half-linear differential equations with periodic coefficients. Ann. Mat. Pura Appl. 190, 395-408 (2011)

10. Došlý, O, Řehák, P: Half-Linear Differential Equations. North-Holland Mathematics Studies, vol. 202. Elsevier, Amsterdam (2005)

11. Fišnarová, S, Mařík, R: On constants in nonoscillation criteria for half-linear differential equations. Abstr. Appl. Anal. 2011, Art. ID 638271 (2011)

12. Sugie, J, Kita, K: Oscillation criteria for second order nonlinear differential equations of Euler type. J. Math. Anal. Appl. 253, 414-439 (2001)

13. Sugie, J, Onitsuka, M: A non-oscillation theorem for nonlinear differential equations with $p$-Laplacian. Proc. R. Soc. Edinb. A 136, 633-647 (2006)

14. Wong, JSW: Oscillation theorems for second-order nonlinear differential equations of Euler type. Methods Appl. Anal. 3, 476-485 (1996)

15. Yamaoka, N: A comparison theorem and oscillation criteria for second-order nonlinear differential equations. Appl. Math. Lett. 23, 902-906 (2010)

16. Agarwal, RP: Difference Equations and Inequalities: Theory, Methods, and Applications, 2nd edn. Monographs and Textbooks in Pure and Applied Mathematics, vol. 228. Dekker, New York (2000)

17. Kelley, W, Peterson, A: Difference Equations: An Introduction with Applications, 2nd edn. Harcourt/Academic Press, San Diego (2001)

doi:10.1186/1687-1847-2012-218

Cite this article as: Yamaoka: Oscillation criteria for second-order nonlinear difference equations of Euler type. Advances in Difference Equations 2012 2012:218

\section{Submit your manuscript to a SpringerOpen ${ }^{\circ}$ journal and benefit from:}

- Convenient online submission

Rigorous peer review

Immediate publication on acceptance

Open access: articles freely available online

- High visibility within the field

- Retaining the copyright to your article 